S. TANNO

KODAI MATH. J.

16 (1993), $171-180$

\title{
THE IDENTITY MAP AS A HARMONIC MAP OF A $(4 r+3)$-SPHERE WITH DEFORMED METRICS
}

Dedicated to Professor Yoji Hatakeyama on his sixtieth birthday

\author{
By SHUKICHI TANNO
}

\section{§ 1. Introduction.}

It is known that the identity map $I d_{M}$ of a compact Riemannian manifold $(M, g)$ is a harmonic map. $(M, g)$ is said to be unstable, if the Jacobi operator $J$ defined by the second variation of the energy functional at $I d_{M}$ has negative eigenvalues. The standard $m$-dimensional sphere $\left(S^{m}, g_{0}\right)$ of constant curvature 1 is unstable for $m \geqq 3$. More generally, unstable, simply connected compact (irreducible) symmetric spaces were determined (Smith [5], Nagano [3], Ohnita [4], Urakawa [11]).

In [9] the author studied instability of spheres $\left(S^{m}, g(t)\right)$ with $m=2 n+1$, as a class of homogeneous Riemannian manifolds which are not symmetric nor Einstein (cf. also Urakawa [12]), and gave the expression of some eigenvector of the Jacobi operator corresponding to a negative eigenvalue. The Riemannian metrics $g(t)$ considered in [9] or [12] is related to the Hopf fibration $\left(S^{2 n+1}, g_{0}\right)$ $\rightarrow\left(C P^{n}, h_{0}\right)$, where $\left(C P^{n}, h_{0}\right)$ denotes the complex projective space with the Fubini-Study metric of constant holomorphic sectional curvature 4 .

As the next step, we study the Riemannian metrics related to the Hopf fibration $\left(S^{4 r+3}, g_{0}\right) \rightarrow\left(Q P^{r}, h_{0}\right)$, where $Q P^{r}$ denotes the quaternion projective space. $\left(S^{4 r+3}, g_{0}\right)$ admits a Sasakian 3-structure $\left\{\eta_{(1)}, \eta_{(2)}, \eta_{(3)}\right\}$. The dual vector fields $\left\{\xi_{(1)}, \xi_{(2)}, \xi_{(3)}\right\}$ define the 3-dimensional distribution on $S^{4 r+3}$ whose integral submanifolds are fibers of the Hopf fibration. We define a 1-parameter family of Riemannian metrics $g(t)$ on $S^{4 r+3}$ by

$$
g(t)=t^{-1} g_{0}+t^{-1}\left(t^{m / 3}-1\right) \Sigma_{\alpha} \eta_{(\alpha)} \otimes \eta_{(\alpha)},
$$

where $m=4 r+3$, and $0<t<\infty$ (cf. Tanno [7]). The volume form for $g(t)$ is unchanged for all $t$. The purpose of this paper is to show the following:

THEOREM. (i) For $m=4 r+3=7,11$, the sphere $\left(S^{4 r+3}, g(t)\right)$ is unstable.

(ii) For $m=4 r+3 \geqq 15$, and for $t \in\left(0, t_{0}(m)\right)$ or $t \in\left(t_{1}(m), \infty\right)$, the sphere $\left(S^{4 r+3}, g(t)\right)$ is unstable, where $t_{0}(m)$ and $t_{1}(m)$ are given in $\S 4$.

(iii) For each eigenfunction $f$ corresponding to the (non-zero) first eigenvalue $\lambda_{1}=4 r+3$ of the Laplacian acting on functions on $\left(S^{4 r+3}, g_{0}\right)$,

Received December 9, 1992. 


$$
\left.f \boldsymbol{\xi}_{(1)}+t^{m / 3} k(t) \nabla \xi_{\operatorname{grad} f} \boldsymbol{\xi}_{(1)}+\left(1-t^{m / 3} k(t)\right)\left(\boldsymbol{\xi}_{(3)} f\right) \boldsymbol{\xi}_{(2)}-\left(\boldsymbol{\xi}_{(2)} f\right) \boldsymbol{\xi}_{(3)}\right)
$$

is an eigenvector corresponding to the negative eigenvalue $\mu(t)$ of the Jacobi operator $J(t) . \quad k(t)$ and $\mu(t)$ are given in $\S 4$. The multiplicity of $\mu(t)$ is $m+1$ for almost all $t$ in $\left(0, t_{0}(m)\right)$ or $\left(t_{1}(m), \infty\right)$.

It is an open problem if the positivity of $\mu(t)$ (for $t_{0}(m)<t<t_{1}(m)$ ) is related to some geometric property, and if $\left(S^{4 r+3}, g(t)\right)$ is stable for $t_{0}(m)<t<t_{1}(m)$.

\section{§2. Preliminaries.}

Let $\left(S^{4 r+3}, g_{0}\right)$ be the unit sphere in the $4(r+1)$-dimensional Euclidean space $E^{4(r+1)}$, where $E^{4(r+1)}$ is considered as a product space $Q \times \cdots \times Q$ of $r+1$ copies of the space $Q$ of quaternions with the canonical metric. Let $\left\{x^{\sigma}, y^{\sigma}, z^{\sigma}, w^{\sigma}\right.$; $\sigma=1, \cdots, r+1)$ be the natural coordinate system of $E^{4(r+1)}$. Let $\{I, J, K\}$ be the quaternion structure of $E^{4(r+1)}$. If one considers a point $x=\left(x^{\sigma}, y^{\sigma}, z^{\sigma}, w^{\sigma}\right)$ of $S^{4 r+3}$ as a unit vector in $E^{4(r+1)}$ and

$$
\begin{aligned}
& I x=\left(y^{\sigma},-x^{\sigma}, w^{\sigma},-z^{\sigma}\right), \\
& J x=\left(z^{\sigma},-w^{\sigma},-x^{\sigma}, y^{\sigma}\right), \\
& K x=\left(w^{\sigma}, z^{\sigma},-y^{\sigma},-x^{\sigma}\right),
\end{aligned}
$$

as tangent vectors at $x$ to $S^{4 r+3}$, we get a field of orthonormal vectors $\left\{\xi_{(1)}\right.$, $\left.\xi_{(2)}, \xi_{(3)}\right\}$ on $S^{4 r+3}$. Each $\xi_{(\alpha)}$ is a Killing vector field on $\left(S^{4 r+3}, g_{0}\right)$, and we have the following:

$$
\left[\xi_{(1)}, \xi_{(2)}\right]=2 \xi_{(3)}, \quad\left[\xi_{(2)}, \xi_{(3)}\right]=2 \xi_{(1)}, \quad\left[\xi_{(3)}, \xi_{(1)}\right]=2 \xi_{(2)} .
$$

The 3-dimensional distribution defined by $\left\{\xi_{(1)}, \xi_{(2)}, \xi_{(3)}\right\}$ on $S^{4 r+3}$ is integrable and each integral submanifold is isometric to a unit 3-sphere in $E^{4}$. This gives the Hopf fibration $S^{4 r+3} \rightarrow Q P^{r}$. Now let $\left\{\eta_{(1)}, \eta_{(2)}, \eta_{(3)}\right\}$ be the dual of $\left\{\xi_{(1)}\right.$, $\left.\xi_{(2)}, \xi_{(3)}\right\}$ with respect to $g_{0}$. Then each $\eta_{(\alpha)}$ defines a contact structure on $S^{4 r+3}$, and $\left\{\eta_{(\alpha)}, g_{0}\right\}$ is a Sasakian structure. Furthermore, $\left\{\eta_{(1)}, \eta_{(2)}, \eta_{(3)} ; g_{0}\right\}$ is called the canonical Sasakian 3-structure of $\left(S^{4 r+3}, g_{0}\right)$. For each $\alpha(\alpha=$ $1,2,3)$, we define a $(1,1)$-tensor field $\phi_{(\alpha)}$ by

$$
\phi_{(\alpha)}=-\nabla \xi_{(\alpha)} .
$$

$\phi_{(1)}, \phi_{(2)}$, and $\phi_{(3)}$ are canonically related to $I, J$, and $K$.

We have the following relations:

$$
\begin{gathered}
\phi_{(\alpha)} \xi_{(\alpha)}=0, \quad \eta_{(\alpha)} \phi_{(\alpha)}=0 \\
\phi_{(\alpha)}^{2} X=-X+\eta_{(\alpha)}(X) \xi_{(\alpha)}, \\
g_{0}(X, Y)=g_{0}\left(\phi_{(\alpha)} X, \phi_{(\alpha)} Y\right)+\eta_{(\alpha)}(X) \eta_{(\alpha)}(Y),
\end{gathered}
$$




$$
\begin{gathered}
\nabla_{\xi(\alpha)} \xi_{(\alpha)}=\nabla_{\xi_{(\alpha)}} \eta_{(\alpha)}=0, \quad L_{\xi_{(\alpha)}} \phi_{(\alpha)}=L_{\xi(\alpha)} \eta_{(\alpha)}=0, \\
\left(\nabla_{X} \phi_{(\alpha)}\right)(Y)=g_{0}(X, Y) \xi_{(\alpha)}-\eta_{(\alpha)}(Y) X, \\
\phi_{(\alpha)} \xi_{(\beta)}=-\phi_{(\beta)} \xi_{(\alpha)}=\xi_{(\gamma)}, \quad[\alpha, \beta, \gamma: \text { cyclic }] \\
\phi_{(\alpha)} \phi_{(\beta)}-\xi_{(\alpha)} \eta_{(\beta)}=-\phi_{(\beta)} \phi_{(\alpha)}+\xi_{(\beta)} \eta_{(\alpha)}=\phi_{(\gamma)}, \quad[\alpha, \beta, \gamma: \text { cyclic }]
\end{gathered}
$$

where $X, Y$ denote tangent vectors or vector fields, and $[\alpha, \beta, \gamma:$ cyclic $]$ means that $\{\alpha, \beta, \gamma\}$ in (2.8) and $(2.9)$ is a cyclic permutation of $(1,2,3)$. This convention is used also in the following. Also, we have the following:

$$
\begin{aligned}
& \eta_{(\alpha)} \phi_{(\beta)}=-\eta_{(\beta)} \phi_{(\alpha)}=\eta_{(\gamma)}, \quad[\alpha, \beta, \gamma: \text { cyclic }] \\
& \nabla_{\xi(\alpha)} \eta_{(\beta)}=-\nabla_{\xi(\beta)} \eta_{(\alpha)}=\eta_{(\gamma)}, \\
& \nabla_{\xi(\alpha)} \xi_{(\beta)}=-\nabla_{\xi(\beta)} \xi_{(\alpha)}=\xi_{(\gamma)}, \quad[\alpha, \beta, \gamma: \text { cyclic }] \\
& L_{\xi(\alpha)} \phi_{(\beta)}=-L_{\xi(\beta)} \phi_{(\alpha)}=2 \phi_{(\gamma)}, \\
& L_{\xi(\alpha)} \eta_{(\beta)}=-L_{\xi(\beta)} \eta_{(\alpha)}=2 \eta_{(\gamma)}, \quad[\alpha, \beta, \gamma: \text { cyclic }]
\end{aligned}
$$

where $L_{X}$ denotes the Lie derivation by $X$. Next, we define an operator $L$ by

$$
L=\Sigma_{\alpha} L_{\xi(\alpha)} L_{\xi(\alpha)} .
$$

The restriction of $L$ (for functions) to each integral submanifold (which is isometric to the 3 -sphere) is identical with the usual Laplacian $\Delta$ acting on functions on $\left(S^{3}, g_{0}\right)$. Thus, we have the following (cf. Tanno [7]):

Proposition 2.1. For a non-negative integer $k$, the eigenspace $V_{k}$ corresponding to the $k$-th eigenvalue of the Laplacian $\Delta$ acting on functions on $\left(S^{4 r+3}, g_{0}\right)$ has the orthogonal decomposition;

$$
V_{k}=W_{k, k}+W_{k, k-2}+\cdots+W_{k, k-2[k / 2]}
$$

such that $f \in W_{k, \theta}$ satisfies

$$
L f=\boldsymbol{\theta}(\boldsymbol{\theta}+2) f .
$$

\section{§3. Riemannian metrics $g(t)$.}

We define $\{g(t) ; 0<t<\infty\}$ such that $g(1)=g_{0}$ on $S^{4 r+3}$ by

$$
g(t)=t^{-1} g_{0}+t^{-1}\left(t^{m / 3}-1\right) \Sigma_{\alpha} \eta_{(\alpha)} \otimes \eta_{(\alpha)} .
$$

For simplicity we denote $g(t)$ by $\tilde{g}$, and $g_{0}$ by $g$ in the following calculation. So, in the local coordinate expression we have

$$
\tilde{g}_{j k}=t^{-1} g_{j k}+t^{-1}\left(t^{m / 3}-1\right) \Sigma_{\alpha} \eta_{(\alpha)_{j}} \eta_{(\alpha)_{k}},
$$




$$
\tilde{g}^{i j}=t g^{i j}-t\left(1-t^{-m / 3}\right) \Sigma_{\alpha} \xi_{(\alpha)}^{i} \xi_{(\alpha)}^{j} .
$$

LEMMA 3.1. The difference $W_{j_{k}}^{i}$ of the Christoffel symbols $\tilde{\Gamma}_{j_{k}}^{i}$ and $\Gamma_{j k}^{i}$ with respect to $g(t)$ and $g_{0}$ is given by

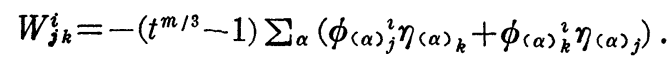

Proof. By a classical formula we have

$$
\begin{aligned}
W_{j k}^{i} & =\tilde{\Gamma}_{j k}^{i}-\Gamma_{j k}^{i} \\
& =(1 / 2) \tilde{g}^{i r}\left(\nabla_{j} \tilde{g}_{r k}+\nabla_{k} \tilde{g}_{r j}-\nabla_{r} \tilde{g}_{j k}\right) .
\end{aligned}
$$

Substituting (3.1) and (3.2) into the above, and using (2.2), etc., we have (3.3).

LEMMA 3.2. The Riemannian curvature tensor $\hat{R}=R_{(t)}$ of $\tilde{g}=g(t)$ is given by

$$
\begin{aligned}
& \tilde{R}_{j k l}^{i}=R_{j k l}^{i}+\left(1-t^{m / 3}\right) \Sigma_{\alpha}\left\{2 \phi_{(\alpha)_{j}^{2}} \phi_{(\alpha)_{k l}}-\phi_{(\alpha)_{l}^{2}} \phi_{(\alpha)_{j k}}+\phi_{(\alpha)_{k}^{2}} \phi_{(\alpha)_{j l}}\right. \\
& \left.+\xi_{(\alpha)}{ }^{i}\left(g_{j k} \eta_{(\alpha)}-g_{j l} \eta_{(\alpha)_{k}}\right)-2 \eta_{(\alpha)_{j}}\left(\delta_{k}^{i} \eta_{(\alpha)_{l}}-\delta_{l}^{i} \eta_{(\alpha)_{k}}\right)\right\} \\
& +\left(1-t^{m / 3}\right)^{2} \sum_{\alpha} \eta_{(\alpha)_{j}}\left(\delta_{k}^{2} \eta_{(\alpha)_{l}}-\delta_{l}^{2} \eta_{(\alpha)_{k}}\right)
\end{aligned}
$$

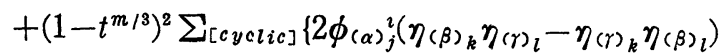

$$
\begin{aligned}
& +\phi_{(\alpha)_{l}}^{i}\left(\eta_{(\beta)_{k}} \eta_{(\gamma)_{j}}-\eta_{(\gamma)_{k}} \eta_{(\beta)_{j}}\right)-\phi_{(\alpha)_{k}}^{i}\left(\eta_{(\beta)_{l}} \eta_{(\gamma)_{j}}-\eta_{(\gamma)_{l}} \eta_{(\beta)_{j}}\right) \\
& \left.+2\left(\xi_{(\alpha)}{ }^{i} \eta_{(\beta)_{j}}-\xi_{(\beta)}{ }^{i} \eta_{(\alpha)_{j}}\right)\left(\eta_{(\alpha)_{k}} \eta_{(\beta)_{l}}-\eta_{(\beta)_{k}} \eta_{(\alpha)_{l}}\right)\right\} \text {. }
\end{aligned}
$$

Proof. We calculate the following:

$$
\widetilde{R}_{j k l}^{i}=R_{j k l}^{i}+\nabla_{k} W_{l j}^{i}-\nabla_{l} W_{k j}^{i}+W_{l j}^{s} W_{k s}^{i}-W_{k j}^{s} W_{l s}^{i} .
$$

By (2.2), (2.7), etc., and (3.3) we have

$$
\begin{aligned}
\nabla_{k} W_{l j}^{i}-\nabla_{l} W_{k j}^{i}= & \left(1-t^{m / 3}\right) \Sigma_{\alpha}\left\{2 \phi_{(\alpha)_{j}^{i}} \phi_{(\alpha)_{k l}}-\phi_{(\alpha)_{l}} \phi_{(\alpha)_{j k}}+\phi_{(\alpha)_{k}{ }_{k}} \phi_{(\alpha)_{j l}}\right. \\
& \left.+\xi_{(\alpha)}{ }^{i}\left(g_{j_{k}} \eta_{(\alpha)_{l}}-g_{j l} \eta_{(\alpha)_{k}}\right)-2 \eta_{(\alpha)_{j}}\left(\delta_{k}^{i} \eta_{(\alpha)_{l}}-\delta_{l}^{i} \eta_{(\alpha)_{k}}\right)\right\} .
\end{aligned}
$$

As for $W_{l j}^{s} W_{k s}^{i}$, we calculate it directly as

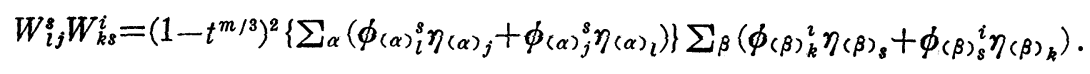

Summing up the results, proof is completed.

LEMMA 3.3. The Ricci curvature tensor $\left(\tilde{R}_{j l}\right)$ and the scalar curvature $\tilde{S}=$ $S_{(t)}$ of $\left(S^{4 r+3}, g(t)\right)$ are given by

$$
\begin{aligned}
\hat{R}_{j l}= & R_{j l}+6\left(1-t^{m / 3}\right) g_{j l} \\
& +\left(1-t^{m / 3}\right)\left[-2 m+(m-3)\left(1-t^{m / 3}\right)\right] \Sigma_{\alpha} \eta_{(\alpha)_{j}} \eta_{(\alpha)_{l}},
\end{aligned}
$$




$$
\tilde{S}=t S-3 t\left(1-t^{-m / 3}\right)\left[2+(m-3) t^{m / 3}\right] \text {. }
$$

The Ricci operator $\tilde{Q}=Q_{(t)}$ is given by

$$
\begin{aligned}
\tilde{R}_{l}^{h}=t & {\left[m-1+6\left(1-t^{m / 3}\right)\right] \delta_{l}^{h} } \\
& -t\left(1-t^{-m / 3}\right)\left[2-(m+3) t^{m / 3}\right] \Sigma_{\alpha} \xi_{(\alpha)}{ }^{n} \eta_{(\alpha)_{l}} .
\end{aligned}
$$

Proof. By contraction $\tilde{R}_{j i l}^{\imath}$ we have $\tilde{R}_{j l} . \quad \tilde{S}$ is given by $\tilde{g}^{j l} \tilde{R}_{j l}$. And, $\hat{R}_{l}^{h}$ $=\tilde{g}^{h j} \hat{R}_{j l}$.

LEMMA 3.4. Each $\xi_{(\alpha)}$ is a Killing vector field with respect to $g(t)$.

Proof. It suffices to show that $\xi_{(1)}$ is a Killing vector field with respect to $g(t) . \quad L_{\xi(1)} g=L_{\xi_{(1)}} \eta_{(1)}=0$ is trivial. By $(2.12)$ we have

$$
\begin{aligned}
& L_{\xi(1)}\left(\eta_{(2)} \otimes \eta_{(2)}\right)=2\left(\eta_{(3)} \otimes \eta_{(2)}+\eta_{(2)} \otimes \eta_{(3)}\right), \\
& L_{\xi(1)}\left(\eta_{(3)} \otimes \eta_{(3)}\right)=-2\left(\eta_{(2)} \otimes \eta_{(3)}+\eta_{(3)} \otimes \eta_{(2)}\right) .
\end{aligned}
$$

Since $g(t)$ is given by (3.1), proof is completed.

We define two operators $\Phi$ and $\Psi$ acting on 1-forms by the following:

$$
\begin{aligned}
& \Phi(w)=\Sigma_{\alpha} \phi_{(\alpha)}^{r s} \nabla_{r} w_{s} \cdot \eta_{(\alpha)}, \\
& \Psi(w)=\Sigma_{(\alpha, \beta, \gamma)} \operatorname{sgn}\left(\begin{array}{lll}
1 & 2 & 3 \\
\alpha & \beta & \gamma
\end{array}\right)\left(\nabla_{\xi(\alpha)} w\right)\left(\xi_{(\beta)}\right) \eta_{(\gamma)},
\end{aligned}
$$

where $\operatorname{sgn}(\cdots)$ denotes the signature of permutation.

Proposition 3.5. The Laplacian $\tilde{\Delta}=\Delta_{(t)}$ for functions and 1-forms are given by

$$
\begin{gathered}
\Delta_{(t)} f=t \Delta f-t\left(1-t^{-m / 3}\right) L f, \\
\Delta_{(t)} w=t \Delta w-t\left(1-t^{-m / 3}\right) L w+2 t\left(1-t^{m / 3}\right) \Phi(w) \\
+2 t\left(1-t^{-m / 3}\right)\left(1-t^{m / 3}\right) \Psi(w) .
\end{gathered}
$$

Proof. (3.8) was proved in [7]. To show (3.9), we calculate the following:

$$
\begin{aligned}
\tilde{\Delta} w_{i}= & \tilde{g}^{r s} \tilde{\nabla}_{r} \tilde{\nabla}_{s} w_{i}-\tilde{R}_{i}^{s} w_{s} \\
= & \tilde{g}^{r s} \tilde{\nabla}_{r}\left(\nabla_{s} w_{i}-W_{s i}^{h} w_{h}\right)-\tilde{R}_{i}^{s} w_{s} \\
= & \tilde{g}^{r s}\left(\nabla_{r} \nabla_{s} w_{i}-\nabla_{r} W_{s i}^{h} w_{h}-W_{s i}^{h} \nabla_{r} w_{h}-W_{r s}^{q} \nabla_{q} w_{\imath}\right. \\
& \left.+W_{r s}^{q} W_{q i}^{h} w_{h}-W_{r i}^{q} \nabla_{s} w_{q}+W_{r i}^{q} W_{s q}^{h} w_{h}\right)-\tilde{R}_{i}^{s} w_{s} .
\end{aligned}
$$

First we check the following relation for each $\alpha$; 


$$
L_{\xi(\alpha)} L_{\xi_{(\alpha)}} w=\nabla_{\xi_{(\alpha)}} \nabla_{\xi(\alpha)} w-2 \nabla_{\xi(\alpha)} w \cdot \phi_{(\alpha)}-w+w\left(\boldsymbol{\xi}_{(\alpha)}\right) \eta_{(\alpha)} .
$$

Then we have

$$
\begin{aligned}
\tilde{g}^{r s} \nabla_{r} \nabla_{s} w_{i}= & t \nabla_{r} \nabla^{r} w_{i}-t\left(1-t^{-m / 3}\right)\left\{3 w_{i}+(L w)_{i}\right. \\
& \left.+\Sigma_{\alpha}\left(2 \nabla_{\xi(\alpha)} w_{h} \cdot \phi_{(\alpha)_{l}}-w\left(\xi_{(\alpha)}\right) \eta_{(\alpha)_{i}}\right)\right\}, \\
\tilde{g}^{r s} \nabla_{r} W_{s i}^{h} w_{h}= & t\left(1-t^{m / 3}\right)\left\{-3 w_{i}+m \Sigma_{\alpha} w\left(\xi_{(\alpha)}\right) \eta_{(\alpha)_{i}}\right\}, \\
\tilde{g}^{r s} W_{s i}^{h} \nabla_{r} w_{h}= & t\left(1-t^{m / 3}\right)\left\{\Sigma_{\alpha} \nabla_{\xi(\alpha)} w_{h} \cdot \phi_{(\alpha)_{i}}-(\Phi(w))_{i}\right\} \\
& -t\left(1-t^{m / 3}\right)\left(1-t^{-m / 3}\right)\left\{\Sigma_{\alpha} \nabla_{\xi_{(\alpha)}} w_{h} \cdot \phi_{(\alpha)_{i}}^{h}+(\Psi(w))_{i}\right\}, \\
\tilde{g}^{r s} W_{r s}^{q} \nabla_{q} w_{i}= & \tilde{g}^{r s} W_{r s}^{q} W_{q i}^{h} w_{h}=0, \\
\tilde{g}^{r s} W_{r i}^{q} W_{s q}^{h} w_{h}= & -3 t\left(1-t^{m / 3}\right)^{2} t^{-m / 3}\left(w_{i}-\sum_{\alpha} w\left(\xi_{(\alpha)}\right) \eta_{(\alpha)}\right) .
\end{aligned}
$$

Summing up the above and using (3.7), we obtain (3.9).

LEMMA 3.6. For each $\alpha, \eta_{(\alpha)}$ is an eigenform of $\Delta_{(t)}$;

$$
\Delta_{(t)} \eta_{(\alpha)}=-2 t\left[(m-1) t^{m / 3}-2\left(1-t^{-m / 3}\right)\left(1+t^{m / 3}\right)\right] \eta_{(\alpha)} .
$$

Proof. It is known that $\Delta \eta_{(\alpha)}=-2(m-1) \eta_{(\alpha)}$ holds. Furthermore we have

$$
\begin{aligned}
& L \eta_{(\alpha)}=-8 \eta_{(\alpha)}, \\
& \Phi\left(\eta_{(\alpha)}\right)=(m-1) \eta_{(\alpha)}, \\
& \Psi\left(\eta_{(\alpha)}\right)=-2 \eta_{(\alpha)} .
\end{aligned}
$$

By (3.9) we obtain (3.11).

Remark. If one wants to obtain geometric expressions of eigen 1-forms of the Laplacian $\Delta_{(t)}$ of $\left(S^{4 r+3}, g(t)\right)$, then the decomposition of $V_{k}$ given by Proposition 2.1 and the expression (3.9) of $\Delta_{(t)}$ are helpful (cf. Tanno [8]).

\section{§4. The Jacobi operator $J_{(t)}$.}

The Jacobi operator $J_{(t)}$ acting on 1 -forms on $\left(S^{4 r+3}, g(t)\right)$ is given by

$$
J_{(t)}=-\Delta_{(t)}-2 Q_{(t)}
$$

and the local coordinate expression is $\left(J_{(t)} w\right)_{i}=-\left(\Delta_{(t)} w\right)_{i}-2 \hat{R}_{\imath}^{h} w_{h}$ (cf. Smith [5]). The Jacobi operator $J_{(t)}$ for vector fields is understood by the natural correspondence between the space of 1 -forms and the space of vector fields. In the following we use $J(t)$ for 1-forms.

Putting $P(w)=\Sigma_{\alpha} w\left(\xi_{(\alpha)}\right) \eta_{(\alpha)}$, we have 
THE IDENTITY MAP AS A HARMONIC MAP OF A $(4 r+3)$-SPHERE

$$
\begin{aligned}
J_{(t)} w= & -t \Delta w+t\left(1-t^{-m / 3}\right) L w-2 t\left(1-t^{m / 3}\right) \Phi(w) \\
& -2 t\left(1-t^{-m / 3}\right)\left(1-t^{m / 3}\right) \Psi(w)-2 t\left[m-1+6\left(1-t^{m / 3}\right)\right] w \\
& +2 t\left(1-t^{-m / 3}\right)\left[2-(m+3) t^{m / 3}\right] P(w) .
\end{aligned}
$$

PROPOSITION 4.1. For each $\alpha$, we have $J_{(t)} \eta_{(\alpha)}=0$.

Proof. This follows from the fact that $\xi_{(\alpha)}$ is a Killing vector field and Killing vector fields belong to the null eigenspace of $J_{(t)}$. Also, the direct calculation using (3.7) and (3.11) is easy.

q. e. d.

Let $V_{1}$ be the eigenspace corresponding to the first eigenvalue $\lambda_{1}=4 r+3$ of the Laplacian acting on functions on $\left(S^{4 r+3}, g(t)\right)$, and let $f \in V_{1}$. Then $f$ satisfies $\nabla_{j} \nabla_{\imath} f=-f g_{\imath}$. Therefore, we have

$$
\begin{aligned}
& \xi_{(\alpha)} \xi_{(\alpha)} f=-f, \\
& \xi_{(\alpha)} \xi_{(\beta)} f=\xi_{(\gamma)} f .
\end{aligned}
$$

LEMMA 4.2. Let $f \in V_{1}$. Then we have the following:

$$
\Delta\left(f \eta_{(1)}\right)=-(3 m-2) f \eta_{(1)}+2 d f \cdot \phi_{(1)},
$$

$$
\begin{aligned}
& L\left(f \eta_{(1)}\right)=-11 f \eta_{(1)}+4\left(\left(\xi_{(3)} f\right) \eta_{(2)}-\left(\xi_{(2)} f\right) \eta_{(3)}\right), \\
& \Phi\left(f \eta_{(1)}\right)=(m-1) f \eta_{(1)}-\left(\left(\xi_{(3)} f\right) \eta_{(2)}-\left(\xi_{(2)} f\right) \eta_{(3)}\right), \\
& \Psi\left(f \eta_{(1)}\right)=-2 f \eta_{(1)}+\left(\left(\xi_{(3)} f\right) \eta_{(2)}-\left(\xi_{(2)} f\right) \eta_{(3)}\right),
\end{aligned}
$$

$$
\Delta\left(\left(\xi_{(3)} f\right) \eta_{(2)}-\left(\xi_{(2)} f\right) \eta_{(3)}\right)=4 f \eta_{(1)}+4 d f \cdot \phi_{(1)}-3 m\left(\left(\xi_{(3)} f\right) \eta_{(2)}-\left(\xi_{(2)} f\right) \eta_{(3)}\right),
$$

(3-ii) $L\left(\left(\xi_{(3)} f\right) \eta_{(2)}-\left(\xi_{(2)} f\right) \eta_{(3)}\right)=8 f \eta_{(1)}-7\left(\left(\xi_{(3)} f\right) \eta_{(2)}-\left(\xi_{(2)} f\right) \eta_{(3)}\right)$,

(3-iii) $\Phi\left(\left(\xi_{(3)} f\right) \eta_{(2)}-\left(\xi_{(2)} f\right) \eta_{(3)}\right)=-2 f \eta_{(1)}+(m-2)\left(\left(\xi_{(3)} f\right) \eta_{(2)}-\left(\xi_{(2)} f\right) \eta_{(3)}\right)$,

(3-iv) $\Psi\left(\left(\xi_{(3)} f\right) \eta_{(2)}-\left(\xi_{(2)} f\right) \eta_{(3)}\right)=2 f \eta_{(1)}-\left(\left(\xi_{(3)} f\right) \eta_{(2)}-\left(\xi_{(2)} f\right) \eta_{(3)}\right)$.

Proof. Verification is done by a direct calculation using (2.2) (2.12).

LEMMA 4.3. With respect to the projection $P$, we have 


$$
P\left(d f \cdot \phi_{(1)}\right)=\left(\xi_{(3)} f\right) \eta_{(2)}-\left(\xi_{(2)} f\right) \eta_{(3)},
$$

$P\left(f \eta_{(1)}\right)=f \eta_{(1)}$ and $P\left(\left(\xi_{(3)} f\right) \eta_{(2)}-\left(\xi_{(2)} f\right) \eta_{(3)}\right)=\left(\xi_{(3)} f\right) \eta_{(2)}-\left(\xi_{(2)} f\right) \eta_{(3)}$.

Proof. The first identity is verified by $(2.8)$.

PROPOSITION 4.4. For $f \in V_{1}, w_{[f, t]}$ is an eigenform of $J_{(t)}$ corresponding to $\mu(t)$ for each $t(0<t<\infty)$, where $w_{[f, t]}$ is defined by

$$
\begin{aligned}
w_{[f, t]}= & f \eta_{(1)}+k(t) d f \cdot \phi_{(1)}+(1-k(t))\left(\left(\xi_{(3)} f\right) \eta_{(2)}-\left(\xi_{(2)} f\right) \eta_{(3)}\right), \\
k(t)= & {\left[2(m-3) t^{m / 3}\right]^{-1}\left\{m+2-2 t^{-m / 3}-6 t^{m / 3}\right.} \\
& \left.+\left[\left(m+2-2 t^{-m / 3}-6 t^{m / 3}\right)^{2}+12(m-3) t^{m / 3}\right]^{1 / 2}\right\},
\end{aligned}
$$

and $\mu(t)$ is given by

$$
\begin{aligned}
\mu(t)= & t\left[-5+t^{-m / 3}+6 t^{m / 3}\right] \\
& -t\left[\left(m+2-2 t^{-m / 3}-6 t^{m / 3}\right)^{2}+12(m-3) t^{m / 3}\right]^{1 / \varepsilon} .
\end{aligned}
$$

For $m=4 r+3=7,11, \mu(t)$ is negative for all $t(0<t<\infty)$. For $m \geqq 15, \mu(t)$ is negative, if $0<t<t_{0}(m)$ or $t_{1}(m)<t$, where

$$
\begin{aligned}
& 3\left(t_{0}(m)\right)^{-m / 3}=2 m-1+[(m-2)(m-14)]^{1 / 2}, \\
& 3\left(t_{1}(m)\right)^{-m / 3}=2 m-1-[(m-2)(m-14)]^{1 / 2} .
\end{aligned}
$$

Proof. We define $w_{[f, t]}$ by (4.5) with undetermined $k(t)$. By (4.2), Lemma 4.2 and Lemma 4.3, we have

$$
\begin{aligned}
J_{(t)} w_{[f, t]}= & t\left\{m-3-t^{-m / 3}-2(m-3) t^{m / 3} k(t)\right\} f \eta_{(1)} \\
& +t\left\{-6-\left(m+7-3 t^{-m / 3}-12 t^{m / 3}\right) k(t)\right\} d f \cdot \phi_{(1)} \\
& +t\left\{m+3-t^{-m / 3}\right. \\
& \left.+\left(m+7-2(m+3) t^{m / 3}-3 t^{-m / 3}\right) k(t)\right\}\left(\left(\boldsymbol{\xi}_{(3)} f\right) \eta_{(2)}-\left(\xi_{(2)} f\right) \eta_{(3)}\right) .
\end{aligned}
$$

Therefore, $J(t) w_{[f, t]}=\mu(t) w_{[f, t]}$ holds, if $k(t)$ is a solution of

$$
(m-3) t^{m / 3} k(t)^{2}-\left(m+2-2 t^{-m / 3}-6 t^{m / 3}\right) k(t)-3=0,
$$

and $\mu(t)$ is given by

$$
\mu(t)=t\left[m-3-t^{-m / 3}-2(m-3) t^{m / 3} k(t)\right] .
$$

Because we are interested in the case where $\mu(t)$ is negative, we choose $k(t)$ as the positive solution of the above equation. So, $k(t)$ is given by (4.6), and consequently, $\mu(t)$ is equal to (4.7). $t$ satisfies $\mu(t)=0$, if and only if 


$$
3 t^{-2 m / 3}-2(2 m-1) t^{-m / 3}+m^{2}+4 m-9=0 .
$$

Therefore, two solutions $t_{0}(m)$ and $t_{1}(m)$ of the above equation are given by (4.8) and (4.9).

Remark. The values $t_{0}(m)$ and $t_{1}(m)$ for $m=15,19$ are given by

$$
\begin{array}{ll}
t_{0}(15)=0.6205 \cdots, & t_{1}(15)=0.6523 \cdots, \\
t_{0}(19)=0.6493 \cdots, & t_{1}(19)=0.7036 \cdots .
\end{array}
$$

PROPOSITION 4.5. Let $\Omega_{(t)}$ denote the eigenspace of $J(t)$ corresponding to $\mu(t)$, and let $\Omega_{(t)}^{\prime}=\left\{w_{[f, t]} ; f \in V_{1}\right\}$. Then $\Omega_{(t)}=\Omega_{(t)}^{\prime}$ and $\operatorname{dim} \Omega_{(t)}=m+1$ hold except for at most countably many values of $t$ in $(0, \infty)$.

Proof. Let $\left\{f_{1}, f_{2}, \cdots, f_{m+1}\right\}$ be a basis of $V_{1}$ and define $w_{\left[f_{\rho}, t\right]}$ by (4.5) with $f=f_{\rho}$. For each $t$, the set

$$
\left\{w_{\left[f_{\rho}, t\right]} ; \rho=1,2, \cdots, m+1\right\}
$$

is linearly independent. In fact, it suffices to see that the set $\left\{w_{\left[f_{\rho}, t\right]}\left(\eta_{(1)}\right)\right\}=$ $\left\{f_{\rho}\right\}$ is linearly independent. So, we have $\operatorname{dim} \Omega_{(t)}^{\prime}=m+1$. For all $t, \Omega_{(t)} \supset \Omega_{(t)}^{\prime}$ is trivial. At $t=1$, we see that $\operatorname{dim} \Omega_{(1)}=m+1=\operatorname{dim} \Omega_{(1)}^{\prime}$. Next, if $t$ is near 1 , then $\Omega_{(t)}=\Omega_{(t)}^{\prime}$, and $\operatorname{dim} \Omega_{(t)}=m+1$. Since $J(t)$ depends on $t$ analytically, the case $\operatorname{dim} \Omega_{(t)}>m+1$ happens only for at most countably many values of $t$.

Proposition 4.6. For each $\alpha, L_{\xi(\alpha)}$ defines an isomorphism of $\Omega_{(t)}^{\prime}$.

Proof. Since $\xi_{(\alpha)}$ is a Killing vector field with respect to $g(t)$, two operators $L_{\xi_{(\alpha)}}$ and $J_{(t)}$ are commutative. Thus, $L_{\xi_{(\alpha)}}$ preserves $\Omega_{(t)}$. Since $\Omega_{(t)}=\Omega_{(t)}^{\prime}$ except for at most countably many values of $t, L_{\xi_{(\alpha)}}$ preserves $\Omega_{(t)}^{\prime}$.

Remark. The expression (4.5) of $w_{[f, t]}$ is based on $\xi_{(1)}$ and $\phi_{(1)}$. Contrary to this, we define $w_{[f, t]}^{\prime}$ and $w_{[f, t]}^{\prime \prime}$ by

$$
\begin{aligned}
& w_{[f, t]}^{\prime}=f \eta_{(2)}+k(t) d f \cdot \phi_{(2)}+(1-k(t))\left(\left(\xi_{(1)} f\right) \eta_{(3)}-\left(\xi_{(3)} f\right) \eta_{(1)}\right), \\
& w_{[f, t]}^{\prime \prime}=f \eta_{(3)}+k(t) d f \cdot \phi_{(3)}+(1-k(t))\left(\left(\xi_{(2)} f\right) \eta_{(1)}-\left(\xi_{(1)} f\right) \eta_{(2)}\right) .
\end{aligned}
$$

Then we have the following relations:

$$
\begin{aligned}
& L_{\xi(1)} w_{[f, t]}=w_{[\xi(1) f, t]}, \\
& L_{\xi(2)} w_{[f, t]}=w_{[\xi(2) f, t]}-2 w_{[f, t]}^{\prime \prime}, \\
& L_{\xi(3)} w_{[f, t]}=w_{[\xi(3) f, t]}+2 w_{[f, t]}^{\prime} .
\end{aligned}
$$

Since $L_{\xi(\alpha)} w_{[f, t]}$ and $w_{[\xi(\alpha) f, t]}$ belong to $\Omega_{(t)}^{\prime}$, we see that $w_{[f, t]}^{\prime}$ and $w_{[f, t]}^{\prime \prime}$ belong to $\Omega_{(t)}^{\prime}$. This means that the expression (4.5) based on $\xi_{(1)}$ and $\phi_{(1)}$ is 
enough for our purpose.

Remark. Let $X$ be a unit tangent vector at a point $x$ of $\left(S^{4 r+3}, g(t)\right)$ satisfying $\eta_{(\alpha)}(X)=0$ for $\alpha=1,2,3$, then the sectional curvature $K_{(t)}\left(X, \phi_{(1)} X\right)$ is given by

$$
K_{(t)}\left(X, \phi_{(1)} X\right)=t\left(4-3 t^{m / 3}\right) .
$$

So, it takes a negative value for $4<3 t^{m / 3}$.

\section{REFERENCES}

[1] P.F. Leung, On the stability of harmonic maps, Lect. Notes in Math., 949, Springer-Verlag, 1982, 122-129.

[2] H. Muto, On the stability of a three-sphere, Kodai Math. Journ., 12 (1989), 109115.

[3] T. NAGANo, Stability of harmonic maps between symmetric spaces, Lect. Notes in Math., 949, Springer-Verlag, 1982, 130-137.

[4] Y. Ohnita, Stability of harmonic maps and standard minimal immersions, Tôhoku Math. Journ., 38 (1986), 259-267.

[5] R. T. Smith, The second variation formula for harmonic mappings, Proc. Amer. Math. Soc., 47 (1975), 229-236.

[6] S. TANno, The first eigenvalue of the Laplacian on spheres, Tôhoku Math. Journ., 31 (1979), 179-185.

[7] S. TANNo, Some metrics on a $(4 r+3)$-sphere and spectra, Tsukuba Journ, Math., 4 (1980), 99-105.

[8] S. TANNo, Geometric expressions of eigen 1-forms of the Laplacian on spheres, Spectra of Riemannian manif. (Kaigai Pub.), Tokyo, 1983, 115-128.

[9] S. TANNO, Instability of spheres with deformed Riemannian metrics, Kodai Math. Journ., 10 (1987), 250-257.

[10] H. URAKAwA, On the least positive eigenvalue of the Laplacian for compact group manifolds, Journ. Math. Soc. Japan, 31 (1979), 209-226.

[11] H. URAKAwA, The first eigenvalue of the Laplacian for positively curved homogeneous Riemannian manifold, Comp. Math., 59 (1986), 57-71.

[12] H. URAKAwA, Stability of harmonic maps and eigenvalues of the Laplacian, Trans. Amer. Math. Soc., 301 (1987), 557-589.

[13] Y.L. XIN, Some results on stable harmonic maps, Duke Math. Journ., 47 (1980), 609-613.

Department of Mathematics

TOKYO INSTITUTE OF TECHNOLOGY

JAPAN 